\title{
Intervención de enfermería en la promoción de salud de las personas en hemodiálisis, una mirada desde la disciplina: Revisión integrativa
}

\author{
Nursing intervention in health promotion for people on hemodialysis, disciplinary \\ perspective: Integrative review
}

\section{Intervenção de enfermagem na promoção da saúde das pessoas em hemodiálise, uma visão da disciplina: Revisão integrativa}

\author{
Nancy Méndez, Barbecho ${ }^{1}$, ORCID 0000-0001-6142-9220 \\ Varinia Rodríguez, Campo ${ }^{2}$, ORCID 0000-0001-8170-6184 \\ Alda Orellana Yáñez ${ }^{3}$, ORCID 0000-0002-2184-0645 \\ Sandra Valenzuela Suazo ${ }^{4}$, ORCID 0000-0002-1308-4835 \\ ${ }^{1}$ Carrera de Enfermería, Universidad de Cuenca. Ecuador \\ $123{ }^{4}$ Facultad de Enfermería, Universidad de Concepción. Chile
}

Recibido: 14/10/2019

Aceptado: 20/03/2020

\begin{abstract}
Resumen: Las personas con Insuficiencia renal crónica terminal en hemodiálisis están susceptibles a complicaciones que pueden prevenirse con promoción de la salud. Estas complicaciones pueden provocar discapacidad e incluso muerte precoz, enfermería forma parte del equipo de salud; razón por la cual, tienen un rol clave en la educación para mejorar la calidad de vida de este grupo de personas. La presente revisión pretende conocer el estado del arte, en relación con la intervención de enfermería en la promoción de la salud en las personas con insuficiencia renal crónica terminal sometidas a hemodiálisis y su impacto en la calidad de vida. Se utilizó la formulación de la pregunta según el acrónimo "PICO" (pacientes, intervención, comparación, resultados); posteriormente, se inició la búsqueda de la información en diferentes bases de datos del repositorio de la Universidad de Concepción: Pub Med, Wef of Science, Biblioteca virtual de Salud, Scielo y Scopus, de manera que se trató de recuperar la mayor cantidad de información; se seleccionaron finalmente siete artículos científicos que cumplieron criterios de inclusión y exclusión. Se evidenció que las intervenciones fueron enfocadas a la alimentación, ingesta de líquidos, empoderamiento, autoeficacia y autocuidado; y una de ellas utilizó la Teoría de rango medio de Nola J. Pender "Modelo de Promoción de Salud". Finalmente, se concluye que la intervención de enfermería en la promoción en este grupo de personas mejora su calidad de vida.
\end{abstract}

Palabras claves: calidad de vida; enfermería; insuficiencia renal crónica; promoción de la salud; revisión integrativa 


\begin{abstract}
People with end-stage renal disease on hemodialysis are susceptible to complications that can be prevented with health promotion, these complications can lead to disability and even early death, nurses are a part of the healthcare team; which is why they have a key role in education to improve the quality of life for this group of people. The present review intends to know the state-of-the-art, in relation to nursing intervention in health promotion for people with end-stage renal disease undergoing hemodialysis and its impact on quality of life. The formulation of the research question was used according to the acronym "PICO" (patients, intervention, comparison, results); subsequently, the search for the information was initiated in different databases of the repository of the University of Concepción: Pub Med, Web of Science, Virtual Health Library, Scielo and Scopus, in attempt to retrieve the greatest amount of information; seven scientific articles that met inclusion and exclusion criteria were finally selected. It was shown that the interventions were focused on diet, fluid intake, empowerment, self-efficacy and self-care; and one of them used Nola J. Pender's middle-range theory "Health Promotion Model". Finally, it concludes that the nursing intervention in promotion in this group of people improves their quality of life.
\end{abstract}

Keywords: quality of life; nursing; chronic renal failure; health promotion; integrative review

Resumo: Pessoas com insuficiência renal crônica terminal em hemodiálise são suscetíveis a complicações que podem ser evitadas com a promoção da saúde. Essas complicações podem causar incapacidade e até morte precoce, a enfermagem faz parte da equipe de saúde; é por isso que eles têm um papel fundamental na educação para melhorar a qualidade de vida desse grupo de pessoas. Esta revisão tem como objetivo conhecer o estado da arte, em relação à intervenção de enfermagem na promoção da saúde em pessoas com insuficiência renal crônica em fase terminal em hemodiálise e seu impacto na qualidade de vida. A questão foi formulada de acordo com a sigla "PICO" (pacientes, intervenção, comparação, resultados); Posteriormente, iniciou-se a busca de informações em diferentes bancos de dados do repositório da Universidade de Concepción: Pub Med, Wef of Science, Biblioteca Virtual em Saúde, Scielo e Scopus, para tentar recuperar a maior quantidade de informações. ; Por fim, foram selecionados sete artigos científicos que atendiam aos critérios de inclusão e exclusão. Evidenciou-se que as intervenções foram focadas em alimentos, ingestão de líquidos, empoderamento, autoeficácia e autocuidado; e um deles usou o "Modelo de Promoção da Saúde" de Nola J. Pender. Por fim, conclui-se que a intervenção de enfermagem na promoção desse grupo de pessoas melhora sua qualidade de vida.

Palavras-chave: qualidade de vida; enfermagem; insuficiência renal crônica; promoção da saúde; revisão integrativa

Correspondencia: Nancy Méndez Barbecho, e-mail: nancy.mendezb@ucuenca.edu.ec 


\section{Introducción}

En el siglo XXI se han experimentado profundos cambios demográficos y epidemiológicos que se traducen en un aumento de la prevalencia e incidencia de las Enfermedades Crónicas no transmisibles (ECNT); estas enfermedades amenazan el desarrollo social, económico, la salud y la vida de millones de personas en el mundo (1). Según la Organización Mundial de Salud (OMS) y la Organización Panamericana de la Salud (OPS) en el 2014, manifiestan que la diabetes y la hipertensión arterial son parte del grupo de ECNT que sumadas al envejecimiento, son los principales factores de riesgo para llevar a las personas a una enfermedad renal crónica (ERC), esto afecta a uno de cada diez adultos en el mundo lo que requiere urgente atención $(1,2)$, provocando consecuencias clínicas, sociales y económicas a las personas enfermas, sus familias y los sistemas de salud (1).

La manifestación más avanzada de la ERC, es la Insuficiencia Renal Crónica Terminal (IRCT) que conlleva a la necesidad de optar por una forma de tratamiento para la sustitución de la función renal, como son diálisis peritonea, hemodiálisis o trasplante (3). La incidencia de nuevos casos en etapas terminales; genera una gran demanda, que actualmente, abarca a más de un millón de pacientes en el mundo que viven gracias a estos métodos de tratamiento(4).

La hemodiálisis parece ser el tratamiento de mayor elección para los pacientes con IRCT, el cual es considerado un proceso seguro, sin embargo, en conjunto con la enfermedad generan alteraciones en la actividad física, provocan estrés, aislamiento social, dependencia e incertidumbre en relación a la salud y al bienestar $(5,6)$; así también, se puede progresar a luto y/o depresión (7), traduciéndose en un sacrificio para los pacientes, sus familiares o los convivientes; especialmente, por la mala Calidad de Vida (CV), las complicaciones, la discapacidad e incluso muerte $\operatorname{precoz}(5,6)$.

La CV según la OMS es considerada como "la percepción que un individuo tiene de su lugar en la existencia, en el contexto de cultura y del sistema de valores en los que vive y en relación con sus expectativas, sus normas y sus inquietudes" (8). La CV de las personas con IRCT se ve afectada sustancialmente en términos de salud física, mental, social, percepción general de salud y carga de la enfermedad, debido a las limitaciones en la dieta, los cambios en la imagen física, en las relaciones sociales, familiares, laborales; y finalmente, porque deben adaptarse a la enfermedad, al tratamiento y a la aparición de problemas físicos, emocionales y espirituales $(9,10,11)$.

Promover la CV en las personas con IRCT en hemodiálisis, se podría considerar como un desafío para desarrollar el empoderamiento de los pacientes en su tratamiento, permitiendo la participación del mismo en su cuidado y en el dominio de su propia vida $(6,12)$, y alcanzar un comportamiento emocional, de adaptación, cognitivo y conductual (7), para enfrentarse con su experiencia de vida. La esencia de Enfermería es el cuidado del ser humano, lo que permite la interacción directa del binomio "enfermera/o - usuaria/o" en casos particulares, como en la IRCT; este enlace es importante en función que permite al personal de enfermería aplicar la estrategia de la promoción de la salud, desde su entendimiento enfermero lo que posteriormente influirá en la CV de las personas enfermas renales (12).

La CV, es una temática de investigación que dentro de la disciplina de enfermería ha sido objeto de su estudio, por lo que el presente trabajo pretende revisar la literatura publicada durante los últimos 18 años, con el objetivo de conocer el estado del arte, respecto a intervenciones de enfermería en promoción de la salud que mejoren la CV en personas con IRCT sometidas a 
hemodiálisis, a manera de evidenciar la importancia de la labor de enfermería como disciplina y profesión, en este grupo de usuarios.

\section{Material y Métodos}

En la presente revisión integrativa, se utilizó la metodología sugerida por la práctica de enfermería basada en la evidencia (EBE), que trata de recoger la mejor evidencia en investigación relacionándola con la expertez clínica (13). Se consideró los tres primeros pasos de Melnyck y Findout-Overholt, los cuáles son:

a. Hacer la pregunta de importancia clínica,

b. Recolección de la mejor evidencia y más relevante,

c. Valorar críticamente la evidencia,

Para formular la pregunta de investigación se utilizó el formato PICO redactándose la pregunta de investigación, de la siguiente manera: ¿Las personas que tienen IRCT sometidas a hemodiálisis, con una intervención de enfermería en promoción de la salud, mejoran la calidad de vida?.

Se realizó una revisión en busca de literatura científica, que respondiera la pregunta planteada; las bases de datos: Pub Med, Wef of Science, Biblioteca virtual de Salud, Scielo y Scopus; para lo cual se utilizaron los siguientes descriptores DeCS y MeSH, tanto en español, inglés y portugués: Promoción de la salud/ Health promotion/ Promoção de Saude - Enfermería/Nursing/Enfermagen - Insuficiencia renal crónica/ Renal insufficiency chronic/Insuficiencia renal crónica - Calidad de Vida/ Quality of life/ Qualidade de Vida); conjugados con el operador booleano AND, por las características de la investigación.

Dentro de los criterios de inclusión se consideraron: estudios primarios cuantitativos; redactados en idioma español, inglés y portugués; publicados entre los años: 2000 y 2018; cuya muestra fueran personas con IRCT sometidas a tratamiento hemodialítico. En los criterios de exclusión se apartaron todas las investigaciones realizadas: en personas menores de 18 años; en diálisis peritoneal o trasplante renal; por otros profesionales que no pertenezcan a la disciplina de enfermería; secundarias como revisiones integrativas, sistemáticas y metaanálisis; estudios de caso; cualitativos y cuyos textos no estén disponible en la web.

Con una compilación inicial de 62 artículos de la literatura científica, se procedió en la primera etapa a aplicar los filtros propios de las bases de datos; para, en una segunda etapa aplicar los criterios de inclusión y exclusión; para finalmente, en una tercera etapa procesar los artículos seleccionados para la revisión (Fig. $\mathrm{N}^{\mathrm{o}} 1$ ). Con relación a las consideraciones éticas, se ha respetado los derechos de autor colocando la cita bibliográfica de cada uno de los artículos utilizados para esta investigación. 
Figura $\mathbf{N}^{\circ}$ 1. Flujograma de revisión de la literatura científica. 2018.

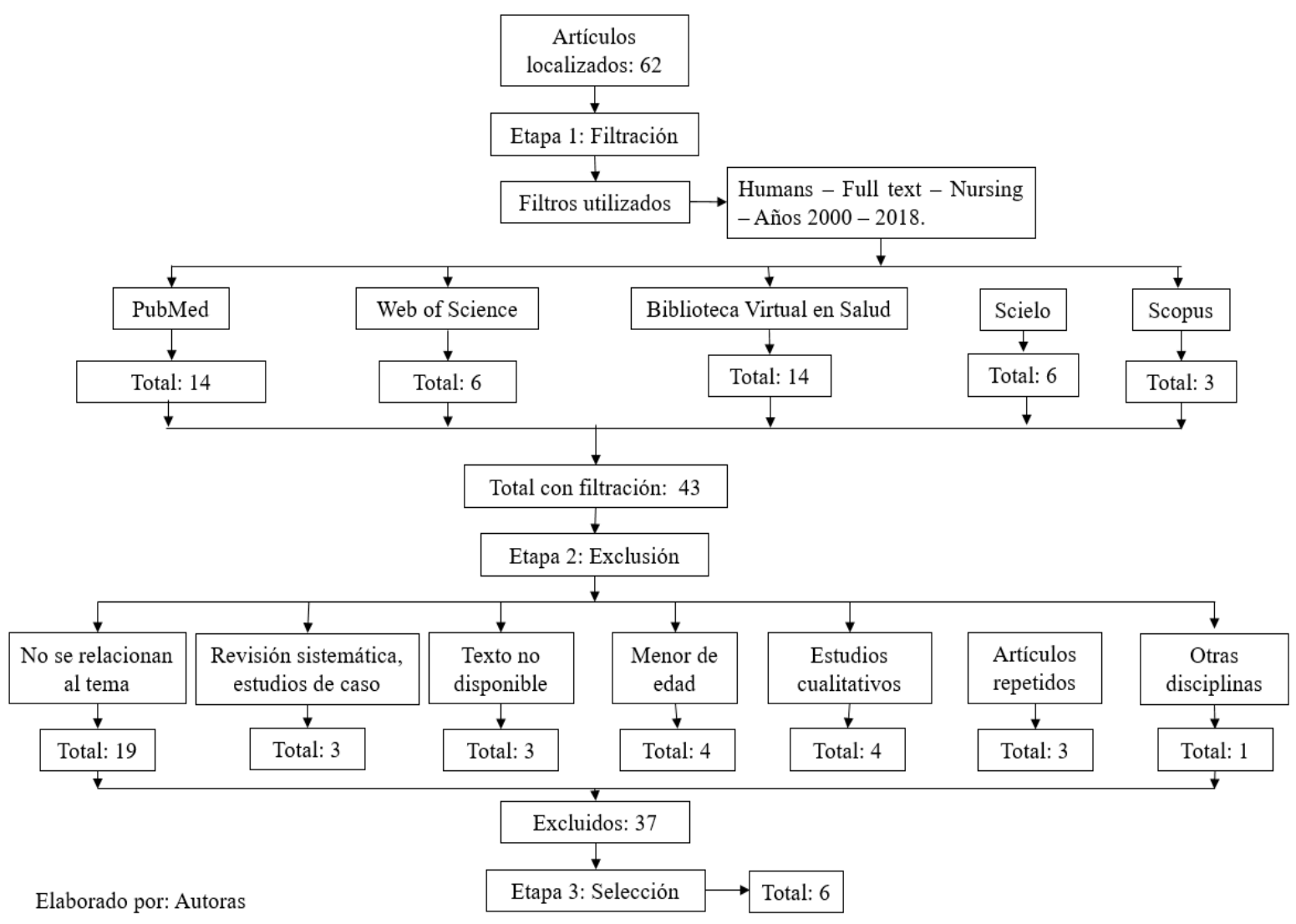

\section{Resultados}

Con los 6 estudios que se relacionan con la temática y que cumplieron con los requisitos propuestos, se procedió a resumirlos (Tabla $\mathrm{N}^{\mathrm{o}} 1$ ) y analizarlos. 
Tabla $\mathbf{N}^{\circ}$ 1. Artículos científicos seleccionados para la revisión. 2018.

\begin{tabular}{|c|c|c|c|c|}
\hline $\begin{array}{l}\text { Título, país y año } \\
\text { de publicación }\end{array}$ & $\begin{array}{c}\text { Diseño de } \\
\text { investigación }\end{array}$ & $\begin{array}{c}\text { Intervención de } \\
\text { enfermería }\end{array}$ & Recolección de datos & Resultados \\
\hline \begin{tabular}{lr}
\multicolumn{2}{l}{ 1.Empowerment } \\
of patients & with \\
end-stage & renal \\
disease & $-\quad$ a \\
randomized & \\
controlled & trial. \\
China 2003(14).
\end{tabular} & $\begin{array}{l}\text { Ensayo } \\
\text { controlado } \\
\text { aleatorizado }\end{array}$ & $\begin{array}{l}\text { Programa de } \\
\text { empoderamiento, } \\
\text { cuidado de la } \\
\text { autoeficacia y de la } \\
\text { depresión en } \\
\text { pacientes con } \\
\text { enfermedad renal en } \\
\text { etapa terminal. }\end{array}$ & \begin{tabular}{ll} 
- & \multicolumn{2}{l}{ Empowerment } \\
Scale (ES). \\
modificada de \\
Anderson. \\
- Strategies used by \\
people to promote \\
health (SUPPH). \\
Beck Depression \\
Inventory (BDI).
\end{tabular} & $\begin{array}{l}\text { Los efectos de la intervención dan como } \\
\text { resultado que los niveles de empoderamiento } \\
\text { en autoeficacia de autocuidado y para } \\
\text { depresión son -significativamente mayores en } \\
\text { el grupo de intervención, por lo cual este grupo } \\
\text { se sentía más capacitado para llevar a cabo su } \\
\text { autocuidado y tenían niveles más bajos de } \\
\text { depresión. }\end{array}$ \\
\hline $\begin{array}{l}\text { 2. A programme to } \\
\text { encourage } \\
\text { participation of } \\
\text { haemodialysis } \\
\text { patients in an } \\
\text { exercise regimen. } \\
\text { Japón } \\
2009(15) \text {. }\end{array}$ & Experimental & $\begin{array}{l}\text { Programa para } \\
\text { pacientes sometidos } \\
\text { a hemodiálisis (HD) } \\
\text { para alentar su } \\
\text { participación } \\
\text { continua en un } \\
\text { régimen } \\
\text { ejercicios. }\end{array}$ & 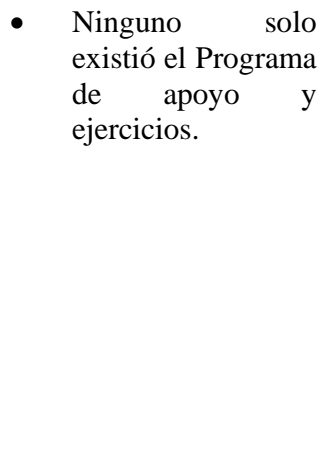 & $\begin{array}{l}\text { Se observó la continuidad de participación de } \\
\text { cada paciente durante el programa de ejercicio. } \\
\text { Todos los } 14 \text { participantes }(100 \%) \text { en el grupo } \\
\text { intervención y } 10 \text { participantes de un total de } \\
16(62.6 \%) \text { en el grupo control participaron } \\
\text { durante las nueve semanas. La prueba exacta } \\
\text { de Fisher se realizó con un nivel de } \\
\text { significación observado de } 0.019 \text {. La } \\
\text { participación continua de los pacientes del } \\
\text { grupo de intervención fue significativamente } \\
\text { mayor que la de los pacientes del grupo } \\
\text { control. }\end{array}$ \\
\hline $\begin{array}{l}\text { 3. Predictors of } \\
\text { dietary and fluid } \\
\text { non-adherence in } \\
\text { Jordanian patients } \\
\text { with end-stage } \\
\text { renal disease } \\
\text { receiving } \\
\text { haemodialysis: a } \\
\text { cross-sectional } \\
\text { study. Jorda-nia } \\
\text { 2012(7). }\end{array}$ & $\begin{array}{l}\text { Diseño } \\
\text { descriptivo, } \\
\text { correlacional } \\
\text { y transversal }\end{array}$ & $\begin{array}{l}\text { Programa educativo } \\
\text { sobre dieta y fluídos } \\
\text { en pacientes } \\
\text { jordanos con } \\
\text { enfermedad renal en } \\
\text { etapa terminal que } \\
\text { reciben hemodiálisis } \\
\text { utilizando el modelo } \\
\text { de promoción de la } \\
\text { salud de Pender. }\end{array}$ & 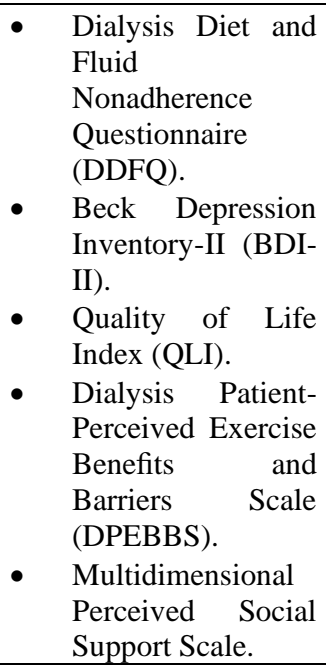 & $\begin{array}{l}\text { El } 27 \% \text { de los pacientes mostraron un } \\
\text { compromiso total con las pautas dietéticas y } \\
23.2 \% \text { a las pautas de fluidos durante los } \\
\text { últimos } 14 \text { días. Usando Pearson } r \text { para } \\
\text { examinar la relación entre variables del estudio } \\
\text { se analiza que la depresión }(M=18.8 \pm 11.4) \\
\text { tuvo un efecto negativo significativo con } \\
\text { dominios de la calidad de vida (con } \\
\text { importancia } r=-0.60, p<0.001 \text {; con } \\
\text { satisfacción, } r=-0.32, p<0.001) \text {. La } \\
\text { aplicación de regresión lineal múltiple reveló } \\
\text { un modelo predictivo de solo dos variables: } \\
\text { edad }(B=-0.22, p=0.05) \text { y función renal } \\
\text { residual }(B=-0.23, p=0.012) \text { para la falta de } \\
\text { adherencia dietética. }\end{array}$ \\
\hline
\end{tabular}


Continuación Tabla $\mathbf{N}^{\circ}$ 1. Artículos científicos seleccionados para la revisión. 2018.

\begin{tabular}{|c|c|c|c|c|}
\hline $\begin{array}{l}\text { 4. Effects of } \\
\text { individualized } \\
\text { exercise program } \\
\text { on physical } \\
\text { function, } \\
\text { psychological } \\
\text { dimensions, and } \\
\text { health - related } \\
\text { quality of life in } \\
\text { patients with } \\
\text { chronic kidney } \\
\text { disease: An } \\
\text { randomized } \\
\text { controlled trial in } \\
\text { China. China } \\
\text { 2016 (16). }\end{array}$ & $\begin{array}{l}\text { Ensayo } \\
\text { controlado } \\
\text { aleatorizado }\end{array}$ & 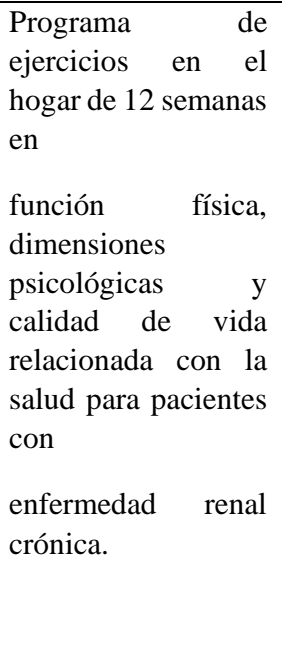 & 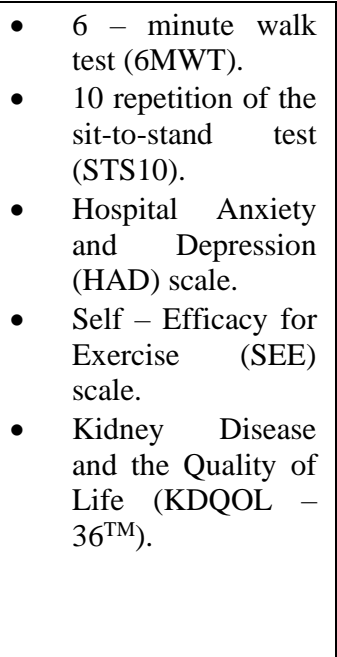 & $\begin{array}{l}\text { Las diferencias entre el grupo de ejercicios y el } \\
\text { grupo control fue estadísticamente } \\
\text { significativo para una distancia de caminata de } \\
6 \text { minutos, el tiempo para completar } 10 \\
\text { repeticiones de la prueba de sentado a pie, } \\
\text { autoeficacia para el ejercicio, la ansiedad y la } \\
\text { depresión, y todo dominios de la calidad de } \\
\text { vida relacionada con la salud después de un } \\
\text { ejercicio de } 12 \text { semanas fue significativo en el } \\
\text { grupo intervención. }\end{array}$ \\
\hline 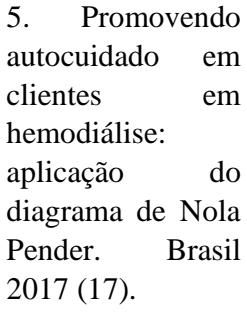 & $\begin{array}{l}\text { Estudio } \\
\text { descriptivo } \\
\text { con diseño } \\
\text { intragrupo. }\end{array}$ & 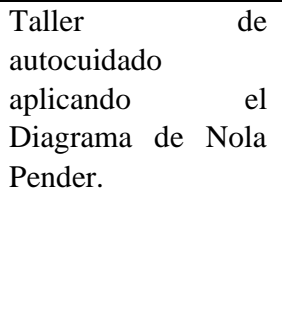 & $\begin{array}{l}\text { Formulario de } \\
\text { solicitud (Consulta } \\
\text { de enfermería } \\
\text { mediada por la } \\
\text { escucha sensible/ } \\
\text { terapéutica). }\end{array}$ & $\begin{array}{l}\text { Las directrices de enfermería llevaron } \\
\text { individuos para adquirir comportamientos de } \\
\text { Modelo de Promoción de Salud con el fin de } \\
\text { satisfacer sus necesidades de bienestar, porque } \\
\text { valoran vivir. El taller estimuló el desarrollo de } \\
\text { la conciencia reflexiva, proporcionando las } \\
\text { condiciones para la reflexión sobre su realidad } \\
\text { y la armonización con la vida. }\end{array}$ \\
\hline $\begin{array}{lr}6 . & \text { Apoyo } \\
\text { educativo y patrón } \\
\text { de vida en el } \\
\text { paciente } \\
\text { tratamiento con } \\
\text { hemodiálisis. } \\
\text { México } \\
2017(18) .\end{array}$ & $\begin{array}{l}\text { Estudio pre- } \\
\text { experimental }\end{array}$ & $\begin{array}{l}\text { Programa educativo } \\
\text { bajo el referente } \\
\text { teórico de Orem con } \\
\text { enfoque en tres } \\
\text { dimensiones: } \\
\text { Responsabilidad en } \\
\text { Salud, Alimentación } \\
\text { e Higiene, consistió } \\
\text { en diez sesiones de } \\
\text { media hora dos } \\
\text { veces por semana } \\
\text { durante la primera } \\
\text { hora de tratamiento } \\
\text { del paciente }\end{array}$ & $\begin{array}{l}\text { - Cuestionario sobre } \\
\text { el patrón de vida } \\
\text { del paciente } \\
\text { tratamiento con } \\
\text { hemodiálisis. }\end{array}$ & $\begin{array}{l}\text { Entre las características se encontró que la } \\
\text { edad promedio de } 36.4 \pm 16.7 \text { años, con igual } \\
\text { distribución tanto para hombres como mujeres } \\
(\mathrm{n}=11) \text {; el } 68.1 \% \text { ( } \mathrm{n}=15) \text { de los pacientes son } \\
\text { económicamente activos, reportando un } \\
\text { tiempo promedio de } 20.6 \pm 15.6 \text { meses de } \\
\text { tratamiento en hemodiálisis. Se obtuvo un } \\
\text { valor de mediana del Patrón de vida } \\
\text { (responsabilidad de vida, higiene y } \\
\text { alimentación) en la fase de pre-intervención de } \\
92 \text { y, de } 107.5 \text { en la fase post-intervención. } \\
\text { Con una diferencia estadísticamente } \\
\text { significativa entre ambas de p<0.001. }\end{array}$ \\
\hline
\end{tabular}

Elaboración propia 
El período de tiempo que se estimó para la búsqueda de artículos para la revisión fue los últimos 18 años, encontrándose dos artículos $(14,15)$ publicados en el periodo 2000 - 2010 y cuatro en los últimos seis años $(7,16,17,18)$.

Cuatro de las investigaciones publicadas se realizaron en el continente asiático $(7,14,15,16)$ y el restante se realizó en Brasil y México $(17,18)$. Respecto a los diseños de las investigaciones cuatro de ellos fueron de tipo experimental $(14,15,16,18)$ y los demás de tipo correlacional o descriptivo $(7,17)$.

Los cuestionarios recolectores de datos de estos estudios fueron variados acorde a los objetivos de cada investigación. Coinciden en dos investigaciones el Inventario de Depresión de Beck en sus versiones BDI (14) y BDI-II (7), y el Cuestionario de Enfermedad Renal y la Calidad de vida en su versión KDQOL-36 (16). Además se utilizan: la Escala de empoderamiento (ES) modificada de Anderson, Estrategias utilizadas por las personas para promover la salud (SUPPH) (14); Cuestionario de dieta de diálisis y no cumplimiento de fluidos (DDFQ), Índice de calidad de vida (QLI), Escala de Beneficios y Barreras del Ejercicio Percibido por el Paciente de Diálisis (DPEBBS), Escala de Apoyo Social Percibido Multidimensional (7); Prueba de caminata 6 minutos (6MWT), 10 repeticiones de la prueba de sentado a pie (STS10), Escala de Depresión y Ansiedad Hospitalaria (HAD), Escala de autoeficacia para el ejercicio (SEE) (16), Formulario de solicitud (Consulta de enfermería mediada por la escucha sensible/ terapéutica) (17); Cuestionario sobre el patrón del vida del paciente con tratamiento de hemodiálisis (18).

Entre los principales resultados de los estudios experimentales cabe destacar que la intervención de enfermería es efectiva en las personas con IRCT sometidas a hemodiálisis, para: el empoderamiento del individuo en la patología y su tratamiento (14), aumentar la autoeficacia (14), el autocuidado (14) y disminuya la depresión (14); el acompañamiento por el personal profesional de enfermería en un régimen de ejercicios en la sala de hemodiálisis permitió que el $100 \%$ de los participantes del grupo experimental no desertarán del programa en relación a la deserción del 62,6\% del grupo control (15); el 27\% de los pacientes mostraron un compromiso total con las pautas dietéticas y $23.2 \%$ a las pautas de fluidos durante los últimos 14 días, se analizó que la depresión tuvo un efecto negativo significativo con dominios de la calidad de vida (7); las dimensiones psicológicas como autoeficacia, ansiedad y depresión, y todos los dominios de la calidad de vida relacionada con la salud después de un ejercicio de doce semanas mejoraron en el grupo intervención (16); enfermería aplica la teoría de rango medio de Nola J. Pender "Modelo de Promoción de la Salud" llevando a los participantes a adquirir comportamientos con el fin de satisfacer sus necesidades de bienestar, porque valoran vivir, con un taller que estimuló el desarrollo de la conciencia reflexiva, proporcionando las condiciones para la reflexión sobre su realidad y la armonización con la vida (17); el apoyo educativo de enfermería sobre el patrón de vida que abarca la responsabilidad de la salud, higiene y alimentación obtuvo un valor de mediana del Patrón de vida (responsabilidad de vida, higiene y alimentación) en la fase de pre-intervención de 92 y de 107.5 en la fase post-intervención, con una diferencia estadísticamente significativa entre ambas de $\mathrm{p}<0.001(18)$.

\section{Discusión}

Los estudios revisados demuestran efectivamente que el empoderamiento en relación a su patología y tratamiento, por medio de una intervención de enfermería, en las personas con IRCT en hemodiálisis, aumenta el autocuidado y la autoeficacia disminuyendo la depresión y mejorando 
la calidad de vida (14).

La mayor parte de las investigaciones se impulsan a partir del año 2012; las intervenciones de enfermería están evidentemente dirigidas a la promoción de la salud para mejorar la CV de las personas en tratamiento hemodialítico; considerando a la actividad física, la alimentación, la ingesta de líquidos, la autoeficacia, y el autocuidado como variables determinantes de una buena CV $(14,15,17,19)$.

Los ejercicios físicos recomendados para este grupo de personas son los aeróbicos de baja intensidad, se debe evitar la utilización del brazo en donde se encuentre localizada la fístula o injerto arterio - venoso, esto disminuye el sedentarismo y aumenta el bienestar (16,20), con lo cual, aumentaría la calidad de vida. Por otro lado, la no adherencia a la dieta y a la ingesta de líquidos se debe a las características individuales de las personas en relación con su cultura y este parámetro del patrón de vida es el más difícil de modificar en los pacientes (7); en base a esta aseveración, se ha demostrado que las intervenciones de enfermería consiguen modificar la autoeficacia y el autocuidado, logrando que las personas se sintieran seguros en el cuidado de su propia salud, disminuyendo significativamente la depresión en ellos (14).

Es importante mencionar que para la disciplina de enfermería la Teoría de mediano rango de Nola J. Pender, "Modelo de Promoción de la Salud" (MPS), fue utilizado en uno de los estudios cuyos autores concluyen que la articulación de la teoría a la práctica permite un vínculo de empatía y confianza en la relación cliente / profesionales de la salud, lo que permite una valiosa posibilidad de interacción para planear en conjunto actividades, acciones y conductas de autocuidado (17), lo que se hace preponderante para la disciplina de enfermería y su rol profesional.

Finalmente, es importante mencionar que en otra investigación se dio a demostrar que el apoyo educativo de enfermería ha generado que todos desarrollen un patrón de vida protector, lo que hace trascendente el papel de enfermería en la promoción de conductas generadoras de salud (18).

\section{Conclusión}

En relación con el planteamiento inicial de esta revisión que fue conocer si la intervención de enfermería promotora en salud mejora la calidad de vida de las personas que tienen IRCT y se encuentran sometidas a hemodiálisis; se concluye que la intervención realizada por los profesionales de enfermería como parte del equipo multidisciplinario de atención en hemodiálisis mejoran la calidad de vida de este grupo de población afectada; por tanto las intervenciones de enfermería son preponderantes para mejorar la CV de las personas con IRCT; sin embargo, es importante recalcar que se debe continuar con la investigación sobre esta línea, para evidenciar mayormente esta intervención a fin de mejorar el estado del arte.

\section{Referencias bibliográficas}

1. Flores J. Enfermedad renal crónica: epidemiología y factores de riesgo. Rev Médica Clínica Las Condes. 2010 Jul;21(4):502-7.

2. Mitchell C. Crece el número de enfermos renales entre los mayores de 60 años con diabetes e hipertensión | OPS OMS [Internet]. Pan American Health Organization / World Health Organization. 2014 [cited 2018 Apr 6]. Available from: http://www.paho.org/hq/index.php?option=com_content\&view=article\&id=9379\%3A2014- 
kidney-disease-rising-among-seniors-diabetes-hypertension\&catid=740\%3Apressreleases $\&$ Itemid $=1926 \&$ lang $=\mathrm{es}$

3. Pérez C, Espadero M, Carmen $\mathbf{M}^{\mathrm{a}}$, Estepa del Árbol M, Crespo Montero R. Factores asociados a calidad de vida relacionada con la salud de pacientes trasplantados de riñón. Enferm Nefrológica. 2015 Sep;18(3):204-26.

4. Pérez Escobar María Mercedes, Herrera Cruz Nuria, Pérez Escobar Elizabeth, Cabrejas Hernández Zenaida. Factores sociales que contribuyen a la morbi-mortalidad en pacientes con Insuficiencia Renal Crónica sometidos a hemodiálisis. Un estudio necesario. Rev Hum Med [Internet]. 2008 [cited 2018 Apr 9]; 8(1). Available from: http://scielo.sld.cu/scielo.php?script=sci_arttext\&pid=S1727-81202008000100007

5. Martín de Francisco A. L El futuro del tratamiento de la enfermedad renal crónica. Nefrología (Madr.) [Internet]. 2010 [cited 2018 Apr 18]; 30(1):1-9 Available from: http://scielo.isciii.es/scielo.php?script=sci_arttext\&pid=S0211-69952010000100001

6. Costa GMA, Pinheiro MBGN, Medeiros SM, Costa RR, Cossi MS. Calidad de vida en pacientes con insuficiencia renal crónica en hemodiálisis. Enferm Glob. 2016 Jul;15(43):5973.

7. Khalil AA, Darawad M, Gamal EA, Hamdan-Mansour AM, Abed MA. Predictors of dietary and fluid non-adherence in Jordanian patients with end-stage renal disease receiving haemodialysis: a cross-sectional study. J Clin Nurs. 22(1-2):127-36.

8. Sofía PK. OMS: Calidad de Vida (2005) [Internet]. [cited 2018 Apr 10]. Available from: http://vidacalida.blogspot.com/2010/12/oms-calidad-de-vida-2005.html

9. Gesualdo GD, Menezes ALC, Rusa SG, Napoleão AA, Figueiredo RM de, Melhado VR, et al. FACTORS ASSOCIATED WITH THE QUALITY OF LIFE OF PATIENTS UNDERGOING HEMODIALYSIS. Texto Amp Contexto - Enferm [Internet]. 2017 [cited 2018 Jun 1];26(2). Available from: http://www.scielo.br/scielo.php?script=sci_abstract\&pid=S010407072017000200338\&lng=en\&nrm=iso\&tlng=en

10. Barros HL, Herazo BY, Aroca MG. Calidad de vida relacionada con la salud en pacientes con enfermedad renal crónica. Rev Fac Med. 2015 Dec 10;63(4):641-7.

11. Badia X. Qué es y cómo se mide la calidad de vida relacionada con la salud. Gastroenterol Hepatol 2004;27(Supl 3):2-6.

12. Bezerra F, Taziana S, Mesquita Lemos A, Sousa C, Maria S, Carvalho L, et al. Promoción de la salud: la calidad de vida en las prácticas de enfermería. Enferm Glob. 2013 Oct;12(32):260-9.

13. Medina EU, Valenzuela CR, Pinto CT, Vidal MR. Enfermería basada en la evidencia: qué es, sus características y dilemas. Investig Educ En Enferm. 2010;28(1):108-18.

14. Tsay S-L, Hung L-O. Empowerment of patients with end-stage renal disease-a randomized controlled trial. Int J Nurs Stud. 2004 Jan;41(1):59-65.

15. Tobita I, Suzuki S, Kobayashi T, Shimizu Y, Umeshita K. A Programme to Encourage Participation of Haemodialysis Patients in an Exercise Regimen. J Ren Care. 35(1):48-53.

16. Tang Q, Yang B, Fan F, Li P, Yang L, Guo Y. Effects of individualized exercise program on physical function, psychological dimensions, and health-related quality of life in patients with chronic kidney disease: A randomized controlled trial in China. Int J Nurs Pract. 2017 Apr;23(2):e12519. 
17. Saldanha BL, Dos Santos I, Costa FV. Promoting self-care in clients on hemodialysis: application of the nola pender's diagram. Rev Pesqui-Cuid E Fundam Online. 2017 Jun;9(2):545-50.

18. Espinoza JAV, Rodríguez RV, Moreno NA, Pérez MG. Apoyo educativo y patrón de vida en el paciente con tratamiento de hemodiálisis. :9.

19. Ma L-C, Chang H-J, Liu Y-M, Hsieh H-L, Lo L, Lin M-Y, et al. The Relationship between Health-Promoting Behaviors and Resilience in Patients with Chronic Kidney Disease [Internet]. The Scientific World Journal. 2013 [cited 2018 May 25]. Available from: https://www.hindawi.com/journals/tswj/2013/124973/

20. Ling K, Wong FSY, Chan W, Chan S, Chan EPY, Cheng Y, et al. Effect of a home exercise program based on tai chi in patients with end-stage renal disease. Perit Dial Int J Int Soc Perit Dial. 2003 Dec;23 Suppl 2:S99-103.

Cómo citar: Méndez Barbecho, N., Rodríguez Campo, V., Orellana Yáñez, A., Valenzuela Suazo, $\mathrm{S}$. Intervención de enfermería en la promoción de salud de las personas en hemodiálisis, una mirada desde la disciplina: Revisión integrativa. Enfermería: Cuidados Humanizados. 2020; 9(1): 54-64. Doi: https://doi.org/10.22235/ech.v9i1.2166

Contribución de los autores: a) Concepción y diseño del trabajo, b) Adquisición de datos, c) Análisis e interpretación de datos, d) Redacción del manuscrito, e) Revisión crítica del manuscrito. N.M.B. ha contribuido en a,b,c,d,e; V.R.C. en a,b,c,d,e; S.L. en a,c,d,e; A.O.Y. en a,c,d,e; S.V.S. en c,d,e.

Editora científica responsable: Dra. Natalie Figueredo 